\title{
Aronson-Bénilan type estimate and the optimal Hölder continuity of weak solutions for the 1- $D$ degenerate Keller-Segel systems
}

Yoshie Sugiyama

\begin{abstract}
We consider the Keller-Segel system of degenerate type $(\mathrm{KS})_{m}$ with $m>1$ below. We establish a uniform estimate of $\partial_{x}^{2} u^{m-1}$ from below. The corresponding estimate to the porous medium equation is well-known as an Aronson-Bénilan type. We apply our estimate to prove the optimal Hölder continuity of weak solutions of $(\mathrm{KS})_{m}$. In addition, we find that the set $D(t):=\{x \in \mathbb{R} ; u(x, t)>0\}$ of positive region to the solution $u$ is monotonically non-decreasing with respect to $t$.
\end{abstract}

\section{Introduction}

We consider the following Keller-Segel system of degenerate type:

$$
(\mathrm{KS})_{m} \begin{cases}\partial_{t} u=\partial_{x}\left(\partial_{x} u^{m}-u^{q-1} \partial_{x} v\right), & x \in \mathbb{R}, t>0 \\ 0=\partial_{x}^{2} v-\gamma v+u, & x \in \mathbb{R}, t>0 \\ u(x, 0)=u_{0}(x), & x \in \mathbb{R}\end{cases}
$$

where $m>1, \gamma>0, q \geq 2 m$. The initial data $u_{0}$ is a non-negative function and in $L^{1} \cap L^{\infty}(\mathbb{R})$ with $u_{0}^{m-1} \in W^{1, \infty}(\mathbb{R})$. This equation is often called as the Keller-Segel model describing the motion of the chemotaxis molds. See e.g., Childress-Percus [8] and Keller-Segel [15].

As we have seen in Sugiyama-Kunii [22], our equation $(\mathrm{KS})_{m}$ has a local weak solution $(u, v)$ under the assumption that $m>1$ and $q \geq 2$. In

2000 Mathematics Subject Classification: 35K65, 35K55, 35B57, 35K45.

Keywords: Parabolic system of degenerate type, Keller-Segel, porous medium, AronsonBénilan estimate, interface, optimal Höder continuity. 
addition, $(\mathrm{KS})_{m}$ has a global weak solution $(u, v)$ on $[0, \infty)$ with the decay property as $t \rightarrow \infty$ if $q \geq m+2$ and if $\left\|u_{0}\right\|_{L^{\frac{q-m}{2}}(\mathbb{R})}$ is small enough.

Concerning the regularity of the weak solution $u$, it is proved in [23] that $\partial_{x} u^{m-1}$ is uniformly bounded on $\mathbb{R} \times(0, T)$. See also Luckhaus-Sugiyama [19] and Sugiyama-Velázquez [25].

The aim of this paper is to show more regularity for $u^{m-1}$ so that $\partial_{x}^{2} u^{m-1}$ is uniformly bounded from below on the region $D_{T}:=\{(x, t) \in$ $\mathbb{R} \times(0, T) ; u(x, t)>0\}$ of positive part of solution $u$. Such an estimate was first obtained by Aronson-Bénilan [4] for the porous medium equation:

$$
\partial_{t} U(x, t)=\partial_{x}^{2} U^{m}(x, t)
$$

So far, there have been a lot of applications of the Aronson-Bénilan type estimate to investigation of solutions of $(\mathrm{PM})_{m}$. See e.g., Aronson [1] and Vázquez [26]. (PM $)_{m}$ may be regarded as the principal part of $(\mathrm{KS})_{m}$. As for $(\mathrm{KS})_{m}$, we shall establish our Aronson-Bénilan type estimate with its applications. Indeed, we show that $u(x, t)$ is Hölder continuous not only in the space variable $x$ but also in the space and time variables $(x, t)$. More precisely, $u^{m-1}(x, t)$ is Hölder continuous with the exponents 1 in $x$ and $\frac{1}{2}$ in $t$, respectively. We expect that this is the optimal Hölder exponent since it coincides with the same optimal one as $(\mathrm{PM})_{m}$. On the other hand, we show that $\partial_{x} u^{m-1+\delta}$ is continuous in both space and time variables for all $\delta>0$. Our result makes it clear that the power $m-1$ to $u$ exhibits the borderline in the sense that $\partial_{x} u^{m-1} \in L^{\infty}(\mathbb{R} \times(0, T))$, while $\partial_{x} u^{m-1+\delta} \in C(\mathbb{R} \times(0, T))$ for all $\delta>0$.

Next, we find that if $u\left(x_{0}, t_{0}\right)>0$ for some $\left(x_{0}, t_{0}\right) \in \mathbb{R} \times(0, T)$, then $x_{0}$ has the property that $u\left(x_{0}, t\right)>0$ for all $t_{0} \leq t<T$. This implies that the support of the weak solution $u(t)$ is monotonically increasing with respect to $t$. Namely, for $D(t):=\{x \in \mathbb{R} ; u(x, t)>0\}$, it holds

$$
D\left(t_{0}\right) \subset D\left(t_{1}\right) \quad \text { for every } 0<t_{0}<t_{1}<T .
$$

In other words, the interface curve $\xi(t)$ has the property of monotonicity in $t \in(0, T)$, where $\xi(t)$ is an expression of the boundary of $D(t)$.

Further application of the Aronson-Bénilan estimate stems from the concrete characterization of the interface curve of $(\mathrm{KS})_{m}$. We see that $(\mathrm{KS})_{m}$ can be rewritten to the following differential equation of the first order:

$$
\partial_{t} u+\partial_{x}(u V)=0
$$

where

$$
V=-\frac{m}{m-1} \partial_{x} u^{m-1}+u^{q-2} \partial_{x} v .
$$


As is well-known, if $V \in C^{1}(\mathbb{R} \times(0, T))$, then the interface curve $\xi(t)$ of $(\mathrm{KS})_{m}$ is determined by the ordinary differential equation

$$
\xi^{\prime}(t)=V(\xi(t), t)
$$

Indeed, we can solve (1.4) with $\xi(s)=x$ uniquely for an arbitrary $x \in \mathbb{R}$, provided $V \in C^{1}(\mathbb{R} \times(0, T))$. Hence the mapping $X_{t, s}(x): x \in \mathbb{R} \mapsto$ $X_{t, s}(x) \equiv \xi(t) \in \mathbb{R}$ is well-defined, which yields the explicit representation of $u$ as

$$
u(x, t)=u_{0}\left(X_{0, t}(x)\right) \exp \left(-\int_{0}^{t} \partial_{x} V\left(X_{s, t}(x), s\right) d s\right) .
$$

This is the well-known method of characteristics for the linear hyperbolic equations of the first order. So, from this representation we see that $u(x, t)=0$ and $u(x, t)>0$ hold if and only if $u_{0}\left(X_{0, t}(x)\right)=0$ and $u_{0}\left(X_{0, t}(x)\right)$ $>0$ hold, respectively. This implies that the mapping $X_{0, t}(x)$ gives the interface curve of $(\mathrm{KS})_{m}$. However, $C^{1}$-regularity of $V$ cannot be expected in the same way as for $(\mathrm{PM})_{m}$. Indeed, $\partial_{x} U^{m-1}$ belongs to $L^{\infty}(\mathbb{R} \times(0, T))$, but not even to $C(\mathbb{R} \times(0, T))$ in general. Therefore, it is not obvious whether $\lim _{x \rightarrow \xi(t)-0} \partial_{x} U^{m-1}(x, t)$ does exist or not.

To overcome such a difficulty, for $(\mathrm{PM})_{m}$, both Aronson [3, page 6] and Knerr [16, Theorem 7.2] introduced the Aronson-Bénilan type estimate, which states a uniform estimate of $\partial_{x}^{2} u^{m-1}$ from below, and proved that $\lim _{x \rightarrow \xi(t)-0} \partial_{x} U^{m-1}(x, t)$ does exist. See Remark 2 (ii) below. For $(\mathrm{KS})_{m}$, our approach to assure the existence of $\lim _{x \rightarrow \xi(t)-0} \partial_{x} u^{m-1}(x, t)$ is similar to that of them and makes it clear that, so far as the case $q \geq 2 m$, their method is applicable to $(\mathrm{KS})_{m}$. It does not seem to be obvious whether the case of $q \geq 2 m$ can be treated as the perturbation from $(\mathrm{PM})_{m}$, and it may be an interesting question whether the case $q=2 m$ exhibits a critical phenomena or not. As a result, we can characterize the interface curve $\xi(t)$ as the solution of the ordinary differential equation (1.4) in the following sense:

$$
\xi^{\prime}(t)=\lim _{x \rightarrow \xi(t)-0} V(x, t)
$$

See [24, Theorem 2.4] for more details.

Throughout this paper, we impose the following assumption:

Assumption. Let the exponents $m>1, q \geq 2 m$ and $\gamma>0$. The initial data $u_{0}$ is a non-negative function satisfying

$$
u_{0} \in L^{1} \cap L^{\infty}(\mathbb{R}) \quad \text { with } \quad u_{0}^{m-1} \in W^{1, \infty}(\mathbb{R}) .
$$


Our definition of a weak solution to $(\mathrm{KS})_{m}$ now reads:

Definition 1 Let the Assumption hold. A pair $(u, v)$ of non-negative functions defined in $\mathbb{R} \times[0, T)$ is called a weak solution of $(\mathrm{KS})_{m}$ on $[0, T)$ if

(i) $u \in L^{\infty}\left(0, T ; L^{1} \cap L^{\infty}(\mathbb{R})\right)$;

(ii) $\partial_{x} u^{m} \in L^{2}\left(0, T ; L^{2}(\mathbb{R})\right)$;

(iii) $v \in L^{\infty}\left(0, T ; H^{1}(\mathbb{R})\right)$;

(iv) $(u, v)$ satisfies the following identities:

$$
\begin{aligned}
& \int_{0}^{T} \int_{\mathbb{R}}\left(\partial_{x} u^{m} \cdot \partial_{x} \varphi-u^{q-1} \partial_{x} v \cdot \partial_{x} \varphi-u \cdot \partial_{t} \varphi\right) d x d t=\int_{\mathbb{R}} u_{0}(x) \varphi(x, 0) d x \\
& \int_{\mathbb{R}}\left(\partial_{x} v \cdot \partial_{x} \psi+\gamma v \psi-u \psi\right) d x=0 \quad \text { a.a. } t \in[0, T)
\end{aligned}
$$

for all $\varphi \in H^{1}\left(0, T ; L^{2}(\mathbb{R})\right) \cap L^{2}\left(0, T ; H^{1}(\mathbb{R})\right)$ with $\varphi(\cdot, T)=0$, and all $\psi \in H^{1}(\mathbb{R})$.

\section{Main Results}

Concerning the time local existence of the weak solution to $(\mathrm{KS})_{m}$, the following result was obtained by the author [22], [23, Proposition 1.1, Theorem 1.2].

Proposition 2.1 ([22],[23]) Let the Assumption hold. There exist $T_{0}$ and a weak solution $(u, v)$ of $(\mathrm{KS})_{m}$ on $\left[0, T_{0}\right)$ with the mass conservation law:

$$
\|u(t)\|_{L^{1}(\mathbb{R})}=\left\|u_{0}\right\|_{L^{1}(\mathbb{R})} \quad \text { for all } 0 \leq t<T_{0},
$$

and with the additional properties:

$$
\begin{aligned}
& u^{m} \in C\left(\left(0, T_{0}\right) ; L_{l o c}^{2}(\mathbb{R})\right) ; \\
& \left(\partial_{x} u^{m}\right)(\cdot, t) \in C(\mathbb{R}) \quad \text { for almost all } t \in\left(0, T_{0}\right) ; \\
& \sup _{0<t<T_{0}}\left\|\partial_{x} u^{m-1}(t)\right\|_{L^{\infty}(\mathbb{R})} \leq C<\infty,
\end{aligned}
$$

where $C=C\left(m, \gamma, q, u_{0}\right)$. Such an interval $T_{0}$ of local existence can be taken as $T_{0}=\left(\left\|u_{0}\right\|_{L^{\infty}(\mathbb{R})}+2\right)^{-q}$, and the weak solution $u(t)$ above satisfies the following estimate:

$$
\|u(t)\|_{L^{\infty}(\mathbb{R})} \leq\left\|u_{0}\right\|_{L^{\infty}(\mathbb{R})}+2 \quad \text { for all } t \in\left[0, T_{0}\right) .
$$

Our first result is on the Hölder continuity of the weak solution $u$ and the continuity of $\partial_{x} u^{m-1+\delta}$ for all $\delta>0$. 
Theorem 2.1 (Hölder continuity) Let the Assumption hold. In addition, we assume that $u_{0}^{\frac{m-1}{2}} \in W^{2, \infty}(\mathbb{R})$ for $1<m<2$ and $u_{0} \in W^{2, \infty}(\mathbb{R})$ for $m \geq 2$, respectively. Suppose that $(u, v)$ is the weak solution of $(\mathrm{KS})_{m}$ on $[0, T)$ given by Proposition 2.1 with $T=T_{0}$. Then, we have the following properties (1) and (2):

(1) There exists a positive constant $C$ depending only on $u_{0}, m, q, \gamma$ such that

$$
\left|u\left(x_{2}, t_{2}\right)-u\left(x_{1}, t_{1}\right)\right| \leq C\left(\left|x_{2}-x_{1}\right|^{\mu}+\left|t_{2}-t_{1}\right|^{\frac{\mu}{2}}\right)
$$

for all $x_{1}, x_{2} \in \mathbb{R}$ and $0<t_{1}, t_{2}<T$, where $\mu:=\min \left\{1, \frac{1}{m-1}\right\}$;

(2) For every $\delta>0, \partial_{x} u^{m-1+\delta}$ is a continuous function on $\mathbb{R} \times(0, T)$ and satisfies the property that $\partial_{x} u^{m-1+\delta}(x, t)=0$ at the point $(x, t) \in \mathbb{R} \times(0, T)$ such that $u(x, t)=0$. Furthermore, in the case of $1<m<2$, we have $\partial_{x} u(x, t)=0$ at the point $(x, t) \in \mathbb{R} \times(0, T)$ such that $u(x, t)=0$.

Remark 1. (i) In our previous paper [23], we found that for each fixed $0<t<T, u(\cdot, t)$ is a Hölder continuous function in the space variable with the same exponent $\mu$ as Theorem 2.1(1) by the fundamental inequality:

$$
|u(x, t)-u(y, t)| \leq\left\{\begin{array}{lc}
\frac{2^{1 / m-1}}{m-1}\|u\|_{L^{\infty}(\mathbb{R} \times(0, T))}^{2-m}\left|u^{m-1}(x, t)-u^{m-1}(y, t)\right|, & 1<m<2, \\
\left|u^{m-1}(x, t)-u^{m-1}(y, t)\right|^{\frac{1}{m-1}}, & m \geq 2
\end{array}\right.
$$

for all $x, y \in \mathbb{R}$ and almost all $0<t<T$. From Theorem 2.1, we obtain more regularity in such a way that $u$ is the Hölder continuous with respect to both space and time variables. Theorem 2.1 says that $u^{m-1}(\cdot, t)$ is Lipshitz continuous in $\mathbb{R}$ with $\partial_{x} u^{m-1} \in L^{\infty}(\mathbb{R} \times(0, T))$. On the other hand, it holds that $\partial_{x} u^{m-1+\delta} \in C(\mathbb{R} \times(0, T))$ for all $\delta>0$.

(ii) The restriction on $q$ such as $q \geq 2 m$ stems from the technical hypothesis for application of the maximum principle to $\partial_{x}^{2} u^{m-1}$. Indeed, to obtain a lower bound of $\partial_{x}^{2} u^{m-1}$, we derive from $(\mathrm{KS})_{m}$ a certain non-linear parabolic equation of the second order with respect to $\partial_{x}^{2} u^{m-1}$, which includes the term $\partial_{x} u^{\frac{q}{2}-1}$ as its coefficient. The application of the maximum principle requires that the coefficients are uniformly bounded in $\mathbb{R} \times(0, T)$. Since we have the only information that $\partial_{x} u^{m-1} \in L^{\infty}(\mathbb{R} \times(0, T))$, it is necessary to assume that $\frac{q}{2}-1 \geq m-1$. This is the technical reason why we impose $q \geq 2 m$.

(iii) The Hölder continuity of solution of $(\mathrm{PM})_{m}$ has been studied intensively for the last 30 years. In this direction, see Aronson [2], Kruzhkov [17], Caffarelli-Friedman [7], Gilding-Peletier [14], Gilding [13], DiBenedetto [10], Bénilan [6], Aronson-Caffarelli [5] and DiBenedetto-Friedman [11]. 
Our second result is on the uniform estimate of $\partial_{x}^{2} u^{m-1}$ from below in a generalized sense.

Theorem 2.2 (Aronson-Bénilan type estimate) Let the Assumption hold. In addition, we assume that $u_{0}^{\frac{m-1}{2}} \in W^{2, \infty}(\mathbb{R})$ for $1<m<2$ and $u_{0} \in$ $W^{2, \infty}(\mathbb{R})$ for $m \geq 2$, restrictively. Suppose that $(u, v)$ is the weak solution of $(\mathrm{KS})_{m}$ on $[0, T)$ given by Proposition 2.1 with $T=T_{0}$. There exists a positive constant $\beta$ depending only on $u_{0}, m, q, \gamma$ such that

$$
\int_{0}^{T} \int_{\mathbb{R}}\left(\beta \varphi-\partial_{x} u^{m-1} \cdot \partial_{x} \varphi\right) d x d t \geq 0
$$

for all $\varphi \in C_{0}^{1}(\mathbb{R} \times(0, T))$ with the property $\varphi(x, t) \geq 0$ for all $(x, t) \in$ $\mathbb{R} \times(0, T)$.

Remark 2. (i) Our estimate (2.6) implies that if $\partial_{x} u^{m-1}(x, t)$ is differentiable in $x$ for some sub-domain $K_{T} \subset \mathbb{R} \times(0, T)$, then it holds

$$
\partial_{x}^{2} u^{m-1}(x, t)>-\beta \quad \text { for all }(x, t) \in K_{T} .
$$

On the other hand, there is a solution $U$ of $(\mathrm{PM})_{m}$ such that

$$
\lim _{h \rightarrow 0} \frac{1}{h}\left(\partial_{x} U^{m-1}\left(x_{0}+h, t_{0}\right)-\partial_{x} U^{m-1}\left(x_{0}-h, t_{0}\right)\right)=+\infty .
$$

This example of $(\mathrm{PM})_{m}$ inspires us that there exists no upper bound of $\partial_{x}^{2} u^{m-1}$ for weak solutions $u$ of $(\mathrm{KS})_{m}$. Hence, such an estimate from below as (2.6) may be reasonable.

(ii) For weak solutions $u$ of $(\mathrm{KS})_{m}$, it is well-known that $u \in C^{\infty}\left(D_{T}\right)$, where $D_{T}:=\{(x, t) \in \mathbb{R} \times(0, T) ; u(x, t)>0\}$. Since $\partial_{x} u^{m-1} \in L^{\infty}(\mathbb{R} \times(0, T))$, it is an interesting question whether or not the $\operatorname{limit}_{\lim } \rightarrow \xi(t)-0 \partial_{x} u^{m-1}(x, t)$ does exist, where $\xi(t)$ denotes the interface curve of $u$, i.e., that the expression of the boundary $\partial D_{T}$ of $D_{T}$ so that $\partial D_{T}=\{(\xi(t), t) ; 0<t<T\}$. Based on (2.6), we see that the function

$$
\partial_{x} u^{m-1}(x, t)+2 \beta x
$$

is monotonically increasing along $x \rightarrow \xi(t)-0$, which guarantees the existence of the limit

$$
\lim _{x \rightarrow \xi(t)-0} \partial_{x} u^{m-1}(x, t) \quad \text { for all } 0<t<T .
$$

This plays an important role in characterization of the interface curve $\xi(t)$ as in (1.5). See Aronson [3, Proof of Theorem, page 6] and Knerr [16, Theorem 7.2] for $(\mathrm{PM})_{m}$.

(iii) Our method for the Aronson-Bénilan type estimate is based on the comparison principle, which is rather technical compared with that in [4]. 
Our third purpose is to show growing-up of the support of the solution $u(t)$ as the time $t$ increases. More precisely, we prove that once the solution $u\left(x_{0}, t_{0}\right)$ of $(\mathrm{KS})_{m}$ becomes positive at some $\left(x_{0}, t_{0}\right) \in \mathbb{R} \times(0, T)$, then $u\left(x_{0}, t\right)$ never vanishes after $t_{0}$.

Theorem 2.3 Let the Assumption hold. In addition, we assume that $u_{0}^{\frac{m-1}{2}} \in$ $W^{2, \infty}(\mathbb{R})$ for $1<m<2$ and $u_{0} \in W^{2, \infty}(\mathbb{R})$ for $m \geq 2$, respectively. Suppose that $(u, v)$ is the weak solution of $(\mathrm{KS})_{m}$ on $[0, T)$ given by Proposition 2.1 with $T=T_{0}$. If $u\left(x_{0}, t_{0}\right)>0$ for some $\left(x_{0}, t_{0}\right) \in \mathbb{R} \times(0, T)$, then the point $x_{0}$ has the property that

$$
u\left(x_{0}, t\right)>0 \quad \text { for all } t_{0}<t<T .
$$

An immediate consequence of Theorem 2.3 is the following corollary.

Corollary 2.4 Under the same hypothesis of Theorem 2.3, the support of the weak solution $u(t)$ given by Proposition 2.1 is monotonically non-decreasing with respect to $t$. Namely, for $D(t):=\{x \in \mathbb{R} ; u(x, t)>0\}$, it holds

$$
D\left(t_{0}\right) \subset D\left(t_{1}\right) \quad \text { for every } 0<t_{0}<t_{1}<T \text {. }
$$

Furthermore, the interface curve $\xi(t)$ of $u$ is either monotone non-decreasing or monotone non-increasing function of $t \in(0, T)$, where $\xi$ is defined in Remark 2 (ii).

Remark 3. In $(\mathrm{PM})_{m}$, a similar property of growing-up of the support of $U(t)$ was obtained, for instance, by Dahlberg-Kenig [9, Theorem 3]. In comparison with $(\mathrm{PM})_{m}$, even in $1-D$ case, $(\mathrm{KS})_{m}$ posses a solution which blows up in a finite time. Hence our Corollary 2.4 seems to be useful to investigate the mass concentration phenomena of blow-up solutions, which will be discussed in a forthcoming paper.

\section{Proof of Theorem 2.1}

\subsection{Approximate equations}

We introduce the following approximating equations of $(\mathrm{KS})_{m}$ :

$$
(\mathrm{KS})_{\varepsilon}\left\{\begin{aligned}
\partial_{t} u_{\varepsilon}(x, t) & =\partial_{x}\left(\partial_{x}\left(u_{\varepsilon}+\varepsilon\right)^{m}-\left(u_{\varepsilon}+\varepsilon\right)^{q-2} u_{\varepsilon} \cdot \partial_{x} v_{\varepsilon}\right) & & (x, t) \in \mathbb{R} \times(0, T), \\
0 & =\partial_{x}^{2} v_{\varepsilon}-\gamma v_{\varepsilon}+u_{\varepsilon}, & & (x, t) \in \mathbb{R} \times(0, T), \\
u_{\varepsilon}(x, 0) & =u_{0 \varepsilon}(x), & & x \in \mathbb{R},
\end{aligned}\right.
$$

where $\varepsilon>0$ is a positive parameter. Let us impose the following assumption on the initial data $u_{0 \varepsilon}$ with $\varepsilon>0$. 
(A.1) $u_{0 \varepsilon}(x) \geq 0$ for all $x \in \mathbb{R}$ and $u_{0 \varepsilon} \in L^{p}(\mathbb{R})$ with

$$
\sup _{0<\varepsilon<1}\left\|u_{0 \varepsilon}\right\|_{L^{p}(\mathbb{R})} \leq\left\|u_{0}\right\|_{L^{p}(\mathbb{R})} \quad \text { for all } p \in[1, \infty],
$$

(i) For $1<m<2$,

$$
\left\|u_{0 \varepsilon}-u_{0}\right\|_{L^{p}(\mathbb{R})} \rightarrow 0 \quad \text { as } \varepsilon \rightarrow 0 \quad \text { for all } p \in\left[\frac{2}{m-1}, \infty\right) ;
$$

(ii) For $m \geq 2$,

$$
\left\|u_{0 \varepsilon}-u_{0}\right\|_{L^{p}(\mathbb{R})} \rightarrow 0 \quad \text { as } \varepsilon \rightarrow 0 \quad \text { for all } p \in[1, \infty)
$$

(A.2) (i) For $1<m<2, \quad u_{0 \varepsilon}^{\frac{m-1}{2}} \in W^{2, \infty}(\mathbb{R})$ with

$$
\sup _{0<\varepsilon<1}\left\|u_{0 \varepsilon}^{\frac{m-1}{2}}\right\|_{W^{2, \infty}(\mathbb{R})} \leq\left\|u_{0}^{\frac{m-1}{2}}\right\|_{W^{2, \infty}(\mathbb{R})}
$$

(ii) For $m \geq 2, \quad u_{0 \varepsilon} \in W^{2, \infty}(\mathbb{R})$ with

$$
\sup _{0<\varepsilon<1}\left\|u_{0 \varepsilon}\right\|_{W^{2, \infty}(\mathbb{R})} \leq\left\|u_{0}\right\|_{W^{2, \infty}(\mathbb{R})}
$$

Remark 4. We can concretely construct $u_{0 \varepsilon}$ satisfying the above (A.1)(A.2)(i) (resp. (A.1)-(A.2)(ii)) by taking

$$
u_{0 \varepsilon}=\left(u_{0}^{\frac{m-1}{2}} * \rho_{\varepsilon}\right)^{\frac{2}{m-1}} \quad\left(\text { resp. } u_{0 \varepsilon}=u_{0} * \rho_{\varepsilon}\right)
$$

with the standard mollifier $\rho_{\varepsilon}$.

When the weak derivatives $\partial_{x} u, \partial_{x}^{2} u$ and $\partial_{t} u$ are in $L^{p}\left(Q_{T}\right)$ for some $p \geq 1$, we say that $u \in W_{p}^{2,1}\left(Q_{T}\right)$, i.e.,

$$
\begin{aligned}
W_{p}^{2,1}\left(Q_{T}\right):=\left\{u \in L^{p}\left(0, T ; W^{2, p}(\mathbb{R})\right) \cap W^{1, p}\left(0, T ; L^{p}(\mathbb{R})\right)\right. & \\
\|u\|_{W_{p}^{2,1}\left(Q_{T}\right)}:= & \|u\|_{L^{p}\left(Q_{T}\right)}+\left\|\partial_{x} u\right\|_{L^{p}\left(Q_{T}\right)} \\
& \left.+\left\|\partial_{x}^{2} u\right\|_{L^{p}\left(Q_{T}\right)}+\left\|\partial_{t} u\right\|_{L^{p}\left(Q_{T}\right)}<\infty\right\}
\end{aligned}
$$

where $Q_{T}=\mathbb{R} \times(0, T)$.

Definition 2 We call $\left(u_{\varepsilon}, v_{\varepsilon}\right)$ a strong solution of $(\mathrm{KS})_{\varepsilon}$ if it belongs to $W_{p}^{2,1} \times W_{p}^{2,1}\left(Q_{T}\right)$ for some $p \geq 1$ and $(\mathrm{KS})_{\varepsilon}$ is satisfied almost everywhere in $Q_{T}$. 
For the strong solution, we consider the case $p=3$ and introduce the space $\mathbf{W}\left(Q_{T}\right)$ defined by

$$
\mathbf{W}\left(Q_{T}\right):=W_{3}^{2,1} \times W_{3}^{2,1}\left(Q_{T}\right) .
$$

The existence of strong solution of $(\mathrm{KS})_{\varepsilon}$ was proved in [20]-[22]. The mass conservation law of $u_{\varepsilon}$ was established in [21, Proposition 7.1].

Proposition 3.1 (local existence of approximating solution) Let $m \geq 1$, $\gamma>0, q \geq 2$. We take $T_{0}:=\left(\left\|u_{0}\right\|_{L^{\infty}(\mathbb{R})}+2\right)^{-q}$. Then, for every $\varepsilon>0$ and every initial data $u_{0 \varepsilon}$ satisfying the hypothesis (A.1)-(A.2), $(\mathrm{KS})_{\varepsilon}$ has the unique non-negative strong solution $\left(u_{\varepsilon}, v_{\varepsilon}\right)$ in $\mathbf{W}\left(Q_{T_{0}}\right)$. Moreover, $u_{\varepsilon}(t)$ satisfies the following a priori estimate

$$
\left\|u_{\varepsilon}(t)\right\|_{L^{\infty}(\mathbb{R})} \leq\left\|u_{0}\right\|_{L^{\infty}(\mathbb{R})}+2 \quad \text { and } \quad \int_{\mathbb{R}} u_{\varepsilon}(x, t) d x=\int_{\mathbb{R}} u_{0 \varepsilon}(x) d x
$$

for all $t \in\left[0, T_{0}\right)$ and all $\varepsilon \in(0,1]$.

It is important to deal with the velocity potential $w_{\varepsilon}$ of $(\mathrm{KS})_{\varepsilon}$, where

$$
w_{\varepsilon}:=\frac{m}{m-1}\left(u_{\varepsilon}+\varepsilon\right)^{m-1} \text {. }
$$

We first obtain a uniform $L^{\infty}$-bound of $\partial_{x} w_{\varepsilon}$.

Lemma 3.1 ([23, Lemma 8.1]) Let the Assumption hold and let $q \geq 2 m$. We assume that $u_{0}^{\frac{m-1}{2}} \in W^{2, \infty}(\mathbb{R})$ for $1<m<2$ and $u_{0} \in W^{2, \infty}(\mathbb{R})$ for $m \geq 2$. For every $\varepsilon>0$, we take $u_{0 \varepsilon}$ so that the hypothesis (A.1)-(A.2) are satisfied. Then the strong solution $u_{\varepsilon}$ of $(\mathrm{KS})_{\varepsilon}$ on $\left[0, T_{0}\right)$ given by Proposition 3.1 has the following property:

$$
\sup _{0<\varepsilon<1}\left(\sup _{0<t<T_{0}}\left\|\partial_{x} w_{\varepsilon}\right\|_{L^{\infty}(\mathbb{R})}\right) \leq C
$$

where $C=C\left(u_{0}, m, q, \gamma\right)$.

Remark 5. (i) It should be noted that the time interval $\left[0, T_{0}\right)$ of the existence of the strong solution $\left(u_{\varepsilon}, v_{\varepsilon}\right)$ can be taken uniformly with respect to $\varepsilon>0$.

(ii) The weak solution $(u, v)$ of $(\mathrm{KS})_{m}$ on $\left[0, T_{0}\right)$ given by Proposition 2.1 can be constructed as the weak limit of $\left(u_{\varepsilon}, v_{\varepsilon}\right)$ as $\varepsilon \rightarrow 0$, where $\left(u_{\varepsilon}, v_{\varepsilon}\right)$ is the strong solution in Proposition 3.1. More precisely, by choosing a 
subsequence of $\left(u_{\varepsilon}, v_{\varepsilon}\right)$ which we denote by $\left(u_{\varepsilon}, v_{\varepsilon}\right)$ itself for simplicity, we have

$$
\begin{array}{cl}
u_{\varepsilon} \rightarrow u & \text { weekly }- \text { star in } L^{\infty}\left(0, T_{0} ; L^{2}(\mathbb{R})\right), \\
u_{\varepsilon}^{m} \rightarrow u^{m} & \text { weekly in } L^{2}\left(0, T_{0} ; H^{1}(\mathbb{R})\right) \text { and strongly in } C\left(\left[0, T_{0}\right) ; L_{l o c}^{2}(\mathbb{R})\right), \\
v_{\varepsilon} \rightarrow v & \text { weekly - star in } L^{\infty}\left(0, T_{0} ; H^{2}(\mathbb{R})\right)
\end{array}
$$

as $\varepsilon \rightarrow 0$. In what follows, we assume that the sequence of approximating solutions $\left(u_{\varepsilon}, v_{\varepsilon}\right)$ satisfies the above convergence.

(iii) The strong solution $\left(u_{\varepsilon}, v_{\varepsilon}\right) \in \mathbf{W}\left(Q_{T_{0}}\right)$ is more regular. Indeed, for every $\varepsilon>0$, it can be shown that $u_{\varepsilon}, v_{\varepsilon} \in C^{\infty}\left(\mathbb{R} \times\left(0, T_{0}\right)\right)$.

We next establish a uniform $L^{\infty}$-bound of $\partial_{x}^{2} w_{\varepsilon}$ from below.

Lemma 3.2 (Aronson-Bénilan type estimate) Let the Assumption hold and let $q \geq 2 m$. We assume that $u_{0}^{\frac{m-1}{2}} \in W^{2, \infty}(\mathbb{R})$ for $1<m<2$ and $u_{0} \in W^{2, \infty}(\mathbb{R})$ for $m \geq 2$. For every $\varepsilon>0$, we take $u_{0 \varepsilon}$ so that the hypothesis (A.1)-(A.2) are satisfied. Then the strong solution $u_{\varepsilon}$ of $(\mathrm{KS})_{\varepsilon}$ on $\left[0, T_{0}\right)$ given by Proposition 3.1 has the following property:

There exists a positive constant $\beta$ depending only on $u_{0}, m, q, \gamma$ but not on $\varepsilon$ such that

$$
\partial_{x}^{2} w_{\varepsilon}(x, t) \geq-\beta
$$

for all $x \in \mathbb{R}$ and $0<t<T$.

Proof of Lemma 3.2. Firstly, multiplying the first equation of $(\mathrm{KS})_{\varepsilon}$ by $m\left(u_{\varepsilon}+\varepsilon\right)^{m-2}$ and then rewriting the result identity by $w_{\varepsilon}$, we have

$$
\begin{aligned}
& \partial_{t} w_{\varepsilon}=(m-1) w_{\varepsilon} \cdot \partial_{x}^{2} w_{\varepsilon}+\left|\partial_{x} w_{\varepsilon}\right|^{2}-\left((q-2)\left(u_{\varepsilon}+\varepsilon\right)^{q-3} u_{\varepsilon}+\left(u_{\varepsilon}+\varepsilon\right)^{q-2}\right) \cdot \partial_{x} v_{\varepsilon} \cdot \partial_{x} w_{\varepsilon} \\
& (3.4)-(m-1)\left(u_{\varepsilon}+\varepsilon\right)^{q-3} u_{\varepsilon} \cdot \partial_{x}^{2} v_{\varepsilon} \cdot w_{\varepsilon} .
\end{aligned}
$$

Differentiating (3.4) once and twice with respect to $x$, we have by (3.2) that

$$
\begin{aligned}
\partial_{t} \partial_{x} w_{\varepsilon}= & (m-1) w_{\varepsilon} \cdot \partial_{x}^{3} w_{\varepsilon}+(m+1) \partial_{x} w_{\varepsilon} \cdot \partial_{x}^{2} w_{\varepsilon} \\
(3.5) & -\left((q-2)\left(u_{\varepsilon}+\varepsilon\right)^{q-3} u_{\varepsilon}+\left(u_{\varepsilon}+\varepsilon\right)^{q-2}\right) \cdot \partial_{x} v_{\varepsilon} \cdot \partial_{x}^{2} w_{\varepsilon}+b_{1}, \\
\partial_{t} \partial_{x}^{2} w_{\varepsilon}= & (m-1) w_{\varepsilon} \cdot \partial_{x}^{4} w_{\varepsilon}+2 m \partial_{x} w_{\varepsilon} \cdot \partial_{x}^{3} w_{\varepsilon} \\
& -\left((q-2)\left(u_{\varepsilon}+\varepsilon\right)^{q-3} u_{\varepsilon}+\left(u_{\varepsilon}+\varepsilon\right)^{q-2}\right) \cdot \partial_{x} v_{\varepsilon} \cdot \partial_{x}^{3} w_{\varepsilon}+(m+1)\left(\partial_{x}^{2} w_{\varepsilon}\right)^{2} \\
(3.6) & +b_{2} \cdot \partial_{x}^{2} w_{\varepsilon}+b_{3}
\end{aligned}
$$


with the estimates

$$
\sup _{0<\varepsilon<1} \sup _{(x, t) \in \mathbb{R} \times[0, T)}\left|b_{i}\left(u_{\varepsilon}, v_{\varepsilon}, \partial_{x} v_{\varepsilon}, \partial_{x} w_{\varepsilon}\right)\right| \leq C, \quad i=1,2,3
$$

for some positive constant $C=C\left(u_{0}, m, q, \gamma\right)$, where $b_{i}=b_{i}\left(u_{\varepsilon}, v_{\varepsilon}, \partial_{x} v_{\varepsilon}, \partial_{x} w_{\varepsilon}\right)$ $(i=1,2,3)$ are functions depending only on $u_{\varepsilon}, v_{\varepsilon}, \partial_{x} v_{\varepsilon}, \partial_{x} w_{\varepsilon}$.

Let $R$ be an arbitrary positive number, and let us take the cut-off function $\eta$ such as

$$
\eta(x):= \begin{cases}1 & \text { for }-2 R+\delta \leq|x|<2 R-\delta, \\ \exp \left(1-\frac{\delta}{2 R-x}\right) & \text { for } 2 R-\delta \leq|x|<2 R \\ 0 & \text { for }|x| \geq 2 R\end{cases}
$$

where $\delta$ is sufficiently small constant. Then, it holds that

$$
\begin{aligned}
&\left|\partial_{x} \eta(x)\right| \leq \frac{c}{\ell^{2} \delta} \cdot \eta(x)^{1-\ell}, \quad\left|\partial_{x}^{2} \eta(x)\right| \leq \frac{c}{\ell^{4} \delta^{2}} \cdot \eta(x)^{1-\ell}, \\
&\left|\partial_{x}^{3} \eta(x)\right| \leq \frac{c}{\ell^{6} \delta^{3}} \cdot \eta(x)^{1-\ell}
\end{aligned}
$$

for all $x \in \mathbb{R}$ and all $0<\ell<1$, where $c$ is an absolute positive constant, which yields that

$$
\begin{aligned}
& \frac{\left(\partial_{x} \eta\right)^{2}}{\eta} \leq \frac{c}{\ell^{4} \delta^{2}} \cdot \eta^{1-2 \ell}, \quad \frac{\partial_{x} \eta \cdot \partial_{x}^{2} \eta}{\eta} \leq \frac{c}{\ell^{6} \delta^{3}} \cdot \eta^{1-2 \ell}, \\
& \frac{\partial_{x} \eta \cdot \partial_{x}^{3} \eta}{\eta} \leq \frac{c}{\ell^{8} \delta^{4}} \cdot \eta^{1-2 \ell}
\end{aligned}
$$

for all $x \in \mathbb{R}$ and all $0<\ell<1$, where $c$ is an absolute positive constant.

Using the above cut-off function $\eta$, we obtain from (3.5), (3.6) and (3.7) that

$$
\begin{aligned}
\partial_{t} \partial_{x}^{2}\left(w_{\varepsilon} \eta\right)= & \left(\partial_{t} \partial_{x}^{2} w_{\varepsilon}\right) \eta+2\left(\partial_{t} \partial_{x} w_{\varepsilon}\right) \partial_{x} \eta+\left(\partial_{t} w_{\varepsilon}\right) \partial_{x}^{2} \eta \\
= & (m-1) w_{\varepsilon}\left(\partial_{x}^{4} w_{\varepsilon}\right) \eta+2(m-1) w_{\varepsilon}\left(\partial_{x}^{3} w_{\varepsilon}\right) \partial_{x} \eta \\
& +\left(2 m \partial_{x} w_{\varepsilon}-\left((q-2)\left(u_{\varepsilon}+\varepsilon\right)^{q-3} u_{\varepsilon}+\left(u_{\varepsilon}+\varepsilon\right)^{q-2}\right) \partial_{x} v_{\varepsilon}\right) \partial_{x}^{3} w_{\varepsilon} \cdot \eta \\
+ & (m+1)\left(\partial_{x}^{2} w_{\varepsilon}\right)^{2} \eta+\left[b_{2} \eta+2(m+1)\left(\partial_{x} w_{\varepsilon}\right) \partial_{x} \eta\right. \\
& -2\left((q-2)\left(u_{\varepsilon}+\varepsilon\right)^{q-3} u_{\varepsilon}+\left(u_{\varepsilon}+\varepsilon\right)^{q-2}\right) \partial_{x} v_{\varepsilon} \cdot \partial_{x} \eta \\
& \left.+(m-1) w_{\varepsilon} \partial_{x}^{2} \eta\right] \partial_{x}^{2} w_{\varepsilon}+b_{0} \partial_{x}^{2} \eta+2 b_{1} \partial_{x} \eta+b_{3} \eta
\end{aligned}
$$

with the estimate

$$
\sup _{0<\varepsilon<1} \sup _{(x, t) \in \mathbb{R} \times[0, T)}\left|b_{0}\left(u_{\varepsilon}, v_{\varepsilon}, \partial_{x} v_{\varepsilon}, \partial_{x} w_{\varepsilon}\right)\right| \leq C
$$

for some positive constant $C=C\left(u_{0}, m, q, \gamma\right)$, where $b_{0}=b_{0}\left(u_{\varepsilon}, v_{\varepsilon}, \partial_{x} v_{\varepsilon}, \partial_{x} w_{\varepsilon}\right)$ is a function depending only on $u_{\varepsilon}, v_{\varepsilon}, \partial_{x} v_{\varepsilon}, \partial_{x} w_{\varepsilon}$. 
On the other hand, it holds that

$$
\begin{aligned}
\left(\partial_{x}^{3} w_{\varepsilon}\right) \eta= & \partial_{x}^{3}\left(w_{\varepsilon} \eta\right)-3\left(\partial_{x}^{2} w_{\varepsilon}\right) \partial_{x} \eta-3\left(\partial_{x} w_{\varepsilon}\right) \partial_{x}^{2} \eta-w_{\varepsilon} \partial_{x}^{3} \eta \\
\left(\partial_{x}^{4} w_{\varepsilon}\right) \eta= & \partial_{x}^{4}\left(w_{\varepsilon} \eta\right)-4\left(\partial_{x}^{3} w_{\varepsilon}\right) \partial_{x} \eta-6\left(\partial_{x}^{2} w_{\varepsilon}\right) \partial_{x}^{2} \eta-4 \partial_{x} w_{\varepsilon} \partial_{x}^{3} \eta-w_{\varepsilon} \partial_{x}^{4} \eta \\
= & \partial_{x}^{4}\left(w_{\varepsilon} \eta\right)-4 \frac{\partial_{x} \eta}{\eta}\left(\partial_{x}^{3}\left(w_{\varepsilon} \eta\right)-3\left(\partial_{x}^{2} w_{\varepsilon}\right) \partial_{x} \eta-3\left(\partial_{x} w_{\varepsilon}\right) \partial_{x}^{2} \eta-w_{\varepsilon} \partial_{x}^{3} \eta\right) \\
& -6\left(\partial_{x}^{2} w_{\varepsilon}\right) \partial_{x}^{2} \eta-4 \partial_{x} w_{\varepsilon} \partial_{x}^{3} \eta-w_{\varepsilon} \partial_{x}^{4} \eta
\end{aligned}
$$

Combining the above identities with (3.10), we have

$$
\begin{aligned}
\partial_{t} \partial_{x}^{2}\left(w_{\varepsilon} \eta\right)= & (m-1) w_{\varepsilon} \partial_{x}^{4}\left(w_{\varepsilon} \eta\right)+2(m-1) w_{\varepsilon} \partial_{x}^{3}\left(w_{\varepsilon} \eta\right) \cdot \frac{\partial_{x} \eta}{\eta} \\
& +\left(2 m \partial_{x} w_{\varepsilon}-\left((q-2)\left(u_{\varepsilon}+\varepsilon\right)^{q-3} u_{\varepsilon}+\left(u_{\varepsilon}+\varepsilon\right)^{q-2}\right) \partial_{x} v_{\varepsilon}\right. \\
& \left.-4(m-1) w_{\varepsilon} \cdot \frac{\partial_{x} \eta}{\eta}\right) \partial_{x}^{3}\left(w_{\varepsilon} \eta\right) \\
& +J_{1}+J_{2} \partial_{x}^{2} w_{\varepsilon}+b_{0} \partial_{x}^{2} \eta+2 b_{1} \partial_{x} \eta+b_{3} \eta \\
& +12(m-1) w_{\varepsilon} \partial_{x} w_{\varepsilon} \frac{\partial_{x} \eta \partial_{x}^{2} \eta}{\eta}+4(m-1) w_{\varepsilon}^{2} \frac{\partial_{x} \eta \partial_{x}^{3} \eta}{\eta}
\end{aligned}
$$

where

$$
\begin{aligned}
J_{1}:= & (m+1)\left(\partial_{x}^{2} w_{\varepsilon}\right)^{2} \eta \\
J_{2}:= & (m-1) w_{\varepsilon}\left(-5 \partial_{x}^{2} \eta+6 \frac{\left(\partial_{x} \eta\right)^{2}}{\eta}\right) \\
& +\left((-4 m+2) \partial_{x} w_{\varepsilon}+\left((q-2)\left(u_{\varepsilon}+\varepsilon\right)^{q-3} u_{\varepsilon}+\left(u_{\varepsilon}+\varepsilon\right)^{q-2}\right) \partial_{x} v_{\varepsilon}\right) \partial_{x} \eta+b_{2} \eta .
\end{aligned}
$$

By (3.1), (3.2) and (3.9), it holds that there exists a positive constant $M_{0}$ depending only on $u_{0}, m, q, \gamma$ such that

$$
J_{2} \leq M_{0}\left(\frac{\eta^{\frac{1}{2}-\ell}}{\ell^{4} \delta^{2}}+\frac{\eta^{\frac{1}{2}-2 \ell}}{\ell^{4} \delta^{2}}+\frac{\eta^{\frac{1}{2}-\ell}}{\ell^{2} \delta}+\eta^{\frac{1}{2}}\right) \eta^{\frac{1}{2}} \leq 4 M_{0} \cdot \frac{1}{\ell^{4} \delta^{2}} \cdot \eta^{\frac{1}{2}}
$$

provided $0<\ell \leq \frac{1}{4}$, which together with the Young inequality yields that

$$
J_{2} \partial_{x}^{2} w_{\varepsilon} \leq \frac{m+1}{4}\left(\partial_{x}^{2} w_{\varepsilon}\right)^{2} \eta+\left(\frac{4 M_{0}}{\ell^{4} \delta^{2}}\right)^{2} .
$$

Thus, from (3.11) together with (3.1), (3.2), (3.9) and (3.12), we find that there exists a positive constant $M_{1}$ depending only on $u_{0}, m, q, \gamma, \delta, \ell$ such that

$$
\begin{aligned}
\partial_{t} \partial_{x}^{2}\left(w_{\varepsilon} \eta\right)> & (m-1) w_{\varepsilon} \partial_{x}^{4}\left(w_{\varepsilon} \eta\right)-M_{1}+\left(2 m \partial_{x} w_{\varepsilon}-\left((q-2)\left(u_{\varepsilon}+\varepsilon\right)^{q-3} u_{\varepsilon}\right.\right. \\
& \left.\left.+\left(u_{\varepsilon}+\varepsilon\right)^{q-2}\right) \partial_{x} v_{\varepsilon}-2(m-1) w_{\varepsilon} \cdot \frac{\partial_{x} \eta}{\eta}\right) \partial_{x}^{3}\left(w_{\varepsilon} \eta\right) .
\end{aligned}
$$


Now let us define the parabolic operator $P$ by

$$
\begin{aligned}
P(h):= & -\partial_{t} h+(m-1) w_{\varepsilon} \cdot \partial_{x}^{2} h-M_{1}+\left(2 m \partial_{x} w_{\varepsilon}\right. \\
& \left.-\left((q-2)\left(u_{\varepsilon}+\varepsilon\right)^{q-3} u_{\varepsilon}+\left(u_{\varepsilon}+\varepsilon\right)^{q-2}\right) \partial_{x} v_{\varepsilon}-2(m-1) w_{\varepsilon} \cdot \frac{\partial_{x} \eta}{\eta}\right) \partial_{x} h .
\end{aligned}
$$

We choose a comparison function $f_{\delta_{*}}(t)$.

$$
\begin{aligned}
f_{\delta_{*}}(t):= & -M_{1}(t+1)+\inf _{x \in(-2 R, 2 R)} \partial_{x}^{2}\left(w_{\varepsilon} \eta\right)(x, 0)+\inf _{s \in(0, T)} \partial_{x}^{2}\left(w_{\varepsilon} \eta\right)\left(-2 R+\delta_{*}, s\right) \\
& +\inf _{s \in(0, T)} \partial_{x}^{2}\left(w_{\varepsilon} \eta\right)\left(2 R-\delta_{*}, s\right), \quad 0<t<T .
\end{aligned}
$$

Here, for a small number $\delta_{*}$ with $0<\delta_{*}<R$, we consider the following domain:

$$
D_{\delta_{*}, T}:=I_{\delta_{*}, R} \times[0, T), \quad I_{\delta_{*}, R}:=\left\{x \in \mathbb{R} ;-2 R+\delta_{*}<x<2 R-\delta_{*}\right\} .
$$

Then, we have

$$
P\left(\partial_{x}^{2}\left(w_{\varepsilon} \eta\right)\right)<0=P\left(f_{\delta_{*}}(t)\right) \text { for all }(x, t) \in D_{\delta_{*}, T} .
$$

In addition, since it holds that

$$
\begin{aligned}
& \partial_{x}^{2}\left(w_{\varepsilon} \eta\right)(x, t)>f_{\delta_{*}}(t) \\
& \quad \text { for all }(x, t) \in \partial D_{\delta_{*}, T} \backslash\left\{(y, T) ;-2 R+\delta_{*} \leq y \leq 2 R-\delta_{*}\right\}
\end{aligned}
$$

by applying the comparison principle to the parabolic operator $P$, e.g., Friedman [12, Theorem 16, page 52] or Lieberman [18, Lemma 9.4], we have

$$
\partial_{x}^{2}\left(w_{\varepsilon} \eta\right)(x, t)>f_{\delta_{*}}(t) \quad \text { for all }(x, t) \in D_{\delta_{*}, T},
$$

which yields that

$$
\partial_{x}^{2} w_{\varepsilon}(x, t)>f_{\delta_{*}}(t) \quad \text { for all }-R<x<R \text {. }
$$

Now, letting $\delta_{*}$ tend to 0 in $(3.13)$, we have

$$
\partial_{x}^{2} w_{\varepsilon}(x, t) \geq-M_{1}(T+1)+\inf _{y \in(-2 R, 2 R)} \partial_{x}^{2}\left(w_{\varepsilon} \eta\right)(y, 0)
$$

for all $-R<x<R$, and $0<t<T$. 
We estimate the term $\inf _{y \in(-2 R, 2 R)} \partial_{x}^{2}\left(w_{\varepsilon} \eta\right)(y, 0)$ independently of $\varepsilon$ from below. Indeed, it holds by (3.8) that

$$
\begin{aligned}
& \quad \inf _{x \in(-2 R, 2 R)} \partial_{x}^{2}\left(w_{\varepsilon}(x, 0) \eta(x)\right) \geq \\
& \geq \inf _{x \in(-2 R, 2 R)}\left(\partial_{x}^{2} w_{\varepsilon}(x, 0)\right) \eta(x) \\
& +\inf _{x \in(-2 R, 2 R)} 2\left(\partial_{x} w_{\varepsilon}(x, 0)\right) \partial_{x} \eta(x)+\inf _{x \in(-2 R, 2 R)} w_{\varepsilon}(x, 0) \partial_{x}^{2} \eta(x) \\
& \geq \inf _{x \in(-2 R, 2 R)}\left(\partial_{x}^{2} w_{\varepsilon}(x, 0)\right) \eta(x)-\frac{c}{\ell^{4} \delta^{2}}\left(\left\|w_{\varepsilon}(\cdot, 0)\right\|_{L^{\infty}(\mathbb{R})}+\left\|\partial_{x} w_{\varepsilon}(\cdot, 0)\right\|_{L^{\infty}(\mathbb{R})}\right)
\end{aligned}
$$

for all $0<\ell<1$, where $c$ is is an absolute positive constant. Since it holds that

$$
\left\|\left(u_{0 \varepsilon}+\varepsilon\right)^{m-1}\right\|_{L^{\infty}(\mathbb{R})} \leq 2^{m-1}\left\|u_{0 \varepsilon}^{m-1}+\varepsilon^{m-1}\right\|_{L^{\infty}(\mathbb{R})} \leq 2^{m-1}\left(\left\|u_{0}\right\|_{L^{\infty}(\mathbb{R})}^{m-1}+1\right),
$$

and since it holds by (A.2) that

$$
\begin{aligned}
& \left\|\partial_{x}\left(u_{0 \varepsilon}+\varepsilon\right)^{m-1}\right\|_{L^{\infty}(\mathbb{R})}=(m-1)\left\|\left(u_{0 \varepsilon}+\varepsilon\right)^{m-2} \partial_{x} u_{0 \varepsilon}\right\|_{L^{\infty}(\mathbb{R})} \\
& \quad \leq \begin{cases}(m-1)\left\|u_{0 \varepsilon}^{m-2} \partial_{x} u_{0 \varepsilon}\right\|_{L^{\infty}(\mathbb{R})} & \text { for } 1<m<2, \\
(m-1) 2^{m-2}\left\|\left(u_{0 \varepsilon}^{m-2}+\varepsilon^{m-2}\right) \partial_{x} u_{0 \varepsilon}\right\|_{L^{\infty}(\mathbb{R})} & \text { for } m \geq 2,\end{cases} \\
& \quad \leq \begin{cases}\left\|\partial_{x} u_{0 \varepsilon}^{m-1}\right\|_{L^{\infty}(\mathbb{R})} & \text { for } 1<m<2, \\
(m-1) 2^{m-2}\left(\left\|u_{0 \varepsilon}^{m-2}+1\right\|_{L^{\infty}(\mathbb{R})}\right)\left\|\partial_{x} u_{0 \varepsilon}\right\|_{L^{\infty}(\mathbb{R})} & \text { for } m \geq 2,\end{cases} \\
& \quad \leq \begin{cases}\left\|\partial_{x} u_{0}^{m-1}\right\|_{L^{\infty}(\mathbb{R})} & \text { for } 1<m<2, \\
(m-1) 2^{m-2}\left(\left\|u_{0}^{m-2}+1\right\|_{L^{\infty}(\mathbb{R})}\right)\left\|\partial_{x} u_{0}\right\|_{L^{\infty}(\mathbb{R})} & \text { for } m \geq 2,\end{cases}
\end{aligned}
$$

we obtain a positive constant $M_{2}$ depending only on $u_{0}, m$ such that

$$
\inf _{x \in(-2 R, 2 R)} \partial_{x}^{2}\left(w_{\varepsilon}(x, 0) \eta(x)\right) \geq \inf _{x \in(-2 R, 2 R)}\left(\partial_{x}^{2} w_{\varepsilon}(x, 0)\right) \eta(x)-\frac{c}{\ell^{4} \delta^{2}} M_{2}
$$

for all $0<\ell<1$, where $c$ is is an absolute positive constant.

In addition, for $1<m<2$, it holds that

$$
\begin{aligned}
& \partial_{x}^{2}\left(u_{0 \varepsilon}+\varepsilon\right)^{m-1}= \\
& =-(m-1)(2-m)\left(u_{0 \varepsilon}+\varepsilon\right)^{m-3}\left(\partial_{x} u_{0 \varepsilon}\right)^{2}+(m-1)\left(u_{0 \varepsilon}+\varepsilon\right)^{m-2} \partial_{x}^{2} u_{0 \varepsilon} \\
& \geq-(m-1)(2-m) u_{0 \varepsilon}^{m-3}\left(\partial_{x} u_{0 \varepsilon}\right)^{2}+\min \left\{0,(m-1) u_{0 \varepsilon}^{m-2} \partial_{x}^{2} u_{0 \varepsilon}\right\}
\end{aligned}
$$

Since it holds that

$$
(m-1) u_{0 \varepsilon}^{m-2} \partial_{x}^{2} u_{0 \varepsilon}=2 u_{0 \varepsilon}^{\frac{m-1}{2}} \cdot \partial_{x}^{2} u_{0 \varepsilon}^{\frac{m-1}{2}}+\frac{(m-1)(3-m)}{2} u_{0 \varepsilon}^{m-3}\left(\partial_{x} u_{0 \varepsilon}\right)^{2},
$$


and since $-(m-1)(2-m)+\frac{(m-1)(3-m)}{2}>0$, we have by (3.16) and (A.2)-(i) that

$$
\begin{aligned}
\partial_{x}^{2}\left(u_{0 \varepsilon}+\varepsilon\right)^{m-1} & \geq-(m-1)(2-m) u_{0 \varepsilon}^{m-3}\left(\partial_{x} u_{0 \varepsilon}\right)^{2} \\
& +\min \left\{0,\left(2 u_{0 \varepsilon}^{\frac{m-1}{2}} \cdot \partial_{x}^{2} u_{0 \varepsilon}^{\frac{m-1}{2}}+\frac{(m-1)(3-m)}{2} u_{0 \varepsilon}^{m-3}\left(\partial_{x} u_{0 \varepsilon}\right)^{2}\right)\right\} \\
& \geq-\frac{4(2-m)}{(m-1)}\left\|\partial_{x} u_{0 \varepsilon}^{\frac{m-1}{2}}\right\|_{L^{\infty}(\mathbb{R})}^{2}-2\left\|u_{0 \varepsilon}^{\frac{m-1}{2}}\right\|_{L^{\infty}(\mathbb{R})}\left\|\partial_{x}^{2} u_{0 \varepsilon}^{\frac{m-1}{2}}\right\|_{L^{\infty}(\mathbb{R})} \\
& \geq-\frac{4(2-m)}{(m-1)}\left\|\partial_{x} u_{0}^{\frac{m-1}{2}}\right\|_{L^{\infty}(\mathbb{R})}^{2}-2\left\|u_{0}^{\frac{m-1}{2}}\right\|_{L^{\infty}(\mathbb{R})}\left\|\partial_{x}^{2} u_{0}^{\frac{m-1}{2}}\right\|_{L^{\infty}(\mathbb{R})} .
\end{aligned}
$$

Thus we obtain that

$$
\begin{aligned}
& \inf _{x \in(-2 R, 2 R)} \partial_{x}^{2}\left(w_{\varepsilon}(x, 0)\right) \eta(x) \geq \\
& \geq-\frac{m}{m-1}\left(\frac{4(2-m)}{(m-1)}\left\|\partial_{x} u_{0}^{\frac{m-1}{2}}\right\|_{L^{\infty}(\mathbb{R})}^{2}+2\left\|u_{0}^{\frac{m-1}{2}}\right\|_{L^{\infty}(\mathbb{R})}\left\|\partial_{x}^{2} u_{0}^{\frac{m-1}{2}}\right\|_{L^{\infty}(\mathbb{R})}\right) .
\end{aligned}
$$

As for the case of $m \geq 2$, we have by (A.2)-(ii) that

$$
\begin{aligned}
\inf _{x \in(-2 R, 2 R)} \partial_{x}^{2}\left(w_{\varepsilon}(x, 0)\right) \eta(x) \geq & \inf _{x \in(-2 R, 2 R)} m(m-2)\left(u_{0 \varepsilon}+\varepsilon\right)^{m-3}\left(\partial_{x} u_{0 \varepsilon}\right)^{2} \eta(x) \\
& \quad+\inf _{x \in(-2 R, 2 R)} m\left(u_{0 \varepsilon}+\varepsilon\right)^{m-2} \partial_{x}^{2} u_{0 \varepsilon} \eta(x) \\
\geq & -m\left\|\left(u_{0 \varepsilon}^{m-2}+1\right)\right\|_{L^{\infty}(-2 R, 2 R)}\left\|\partial_{x}^{2} u_{0 \varepsilon}\right\|_{L^{\infty}(-2 R, 2 R)} \\
& \geq-m\left\|\left(u_{0}^{m-2}+1\right)\right\|_{L^{\infty}(\mathbb{R})}\left\|\partial_{x}^{2} u_{0}\right\|_{L^{\infty}(\mathbb{R})} .
\end{aligned}
$$

Thus we find by (3.15), (3.17) and (3.18) that there exists a positive constant $M_{3}$ depending only on $u_{0}, m$ such that

$$
\inf _{x \in(-2 R, 2 R)} \partial_{x}^{2}\left(w_{\varepsilon}(x, 0)\right) \eta(x) \geq-M_{3}-\frac{c}{\ell^{4} \delta^{2}} M_{2}
$$

for all $0<\ell<1$, where $c$ is is an absolute positive constant. Combining (3.14) with (3.19) and taking $\ell=\frac{1}{4}$, we have

$$
\partial_{x}^{2} w_{\varepsilon}(x, t) \geq-M_{1}(T+1)-M_{3}-\frac{4^{4} c}{\delta^{2}} M_{2}=: \beta
$$

for all $-R<x<R$, and $0<t<T$, where $\beta$ is a positive constant independent of $0<\varepsilon<1$. Since $\beta$ is independent of $R$, we establish (3.3). Thus we complete the proof of Lemma 3.2. 


\subsection{Proof of Theorem 2.1}

Proof of Theorem 2.1(1). We apply a similar argument to that in Caffarelli-Friedman [7, page 111, Remark 1]. Using (3.1), (3.2) and (3.3), we shall show $(2.5)$. We have by $(\mathrm{KS})_{\varepsilon}$ that

$$
\partial_{t}\left(\left(u_{\varepsilon}(x, t)+\varepsilon\right)^{m-1}\right) \geq-C\left(u_{\varepsilon}(x, t)+\varepsilon\right)^{m-1}
$$

for all $x \in \mathbb{R}$ and $0<t<T$ with some positive constant $C=C\left(u_{0}, m, q, \gamma\right)$ independent of $0<\varepsilon<1$. Indeed, it holds that

$$
\begin{aligned}
\partial_{t}\left(u_{\varepsilon}+\varepsilon\right)^{m-1} & =(m-1)\left(u_{\varepsilon}+\varepsilon\right)^{m-2} \partial_{t} u_{\varepsilon} \\
& =(m-1)\left(u_{\varepsilon}+\varepsilon\right)^{m-2}\left(\partial_{x}^{2}\left(u_{\varepsilon}+\varepsilon\right)^{m}-\partial_{x}\left(\left(u_{\varepsilon}+\varepsilon\right)^{q-2} u_{\varepsilon} \cdot \partial_{x} v_{\varepsilon}\right)\right) .
\end{aligned}
$$

It is easily seen that

$$
\begin{aligned}
& \partial_{x}^{2}\left(u_{\varepsilon}+\varepsilon\right)^{m}=\frac{m}{m-1}\left(u_{\varepsilon}+\varepsilon\right) \cdot \partial_{x}^{2}\left(u_{\varepsilon}+\varepsilon\right)^{m-1}+m\left(u_{\varepsilon}+\varepsilon\right)^{m-2}\left(\partial_{x} u_{\varepsilon}\right)^{2} \\
& \geq-\frac{m}{m-1}\left(u_{\varepsilon}+\varepsilon\right) \cdot \partial_{x}^{2}\left(u_{\varepsilon}+\varepsilon\right)^{m-1} \\
& \begin{aligned}
& \geq-\frac{m \beta}{m-1}\left(u_{\varepsilon}+\varepsilon\right), \\
&3.23) \quad \\
&-\partial_{x}\left(\left(u_{\varepsilon}+\varepsilon\right)^{q-2} u_{\varepsilon} \cdot \partial_{x} v_{\varepsilon}\right)=-\frac{q-2}{m-1}\left(u_{\varepsilon}+\varepsilon\right)^{q-m-1} u_{\varepsilon} \cdot \partial_{x} v_{\varepsilon} \cdot \partial_{x}\left(u_{\varepsilon}+\varepsilon\right)^{m-1} \\
&-\frac{1}{m-1}\left(u_{\varepsilon}+\varepsilon\right)^{q-m} \cdot \partial_{x} v_{\varepsilon} \cdot \partial_{x}\left(u_{\varepsilon}+\varepsilon\right)^{m-1} \\
&-\left(u_{\varepsilon}+\varepsilon\right)^{q-2} u_{\varepsilon} \cdot \gamma v_{\varepsilon}+\left(u_{\varepsilon}+\varepsilon\right)^{q-2}\left(u_{\varepsilon}\right)^{2} \\
& \geq-C\left(u_{\varepsilon}+\varepsilon\right)
\end{aligned}
\end{aligned}
$$

for some positive constant $C=C\left(u_{0}, m, q, \gamma\right)$ independent of $0<\varepsilon<1$, where $\beta$ is the same one as in (3.3). Substituting (3.23) and (3.24) into (3.22), we obtain (3.21).

We now integrate (3.21) with respect to the time variable, which with the aid of (3.1) yields that

$$
\left(u_{\varepsilon}\left(x, t_{2}\right)+\varepsilon\right)^{m-1} \geq\left(u_{\varepsilon}\left(x, t_{1}\right)+\varepsilon\right)^{m-1}-C_{1}\left|t_{2}-t_{1}\right|
$$

for all $x \in \mathbb{R}$ and $0<t_{1}<t_{2}<T$ with some positive constant $C_{1}=$ $C_{1}\left(u_{0}, m, q, \gamma\right)$ independent of $0<\varepsilon<1$. On the other hand, it holds by (3.2) with $T=T_{0}$ that

$$
\left.\mid u_{\varepsilon}\left(x_{1}, t_{1}\right)+\varepsilon\right)^{m-1}-\left(u_{\varepsilon}\left(x_{2}, t_{1}\right)+\varepsilon\right)^{m-1}\left|\leq C_{2}\right| x_{2}-x_{1} \mid
$$


for all $x_{1}, x_{2} \in \mathbb{R}$ and $0<t_{1}<T$ with some positive constant $C_{2}=$ $C_{2}\left(u_{0}, m, q, \gamma\right)$ independent of $0<\varepsilon<1$. As a result, combining (3.25) with (3.26), we obtain that

$$
\left(u_{\varepsilon}\left(x_{2}, t_{2}\right)+\varepsilon\right)^{m-1} \geq\left(u_{\varepsilon}\left(x_{1}, t_{1}\right)+\varepsilon\right)^{m-1}-\left(C_{1}+C_{2}\right)\left(\left|t_{2}-t_{1}\right|+\left|x_{2}-x_{1}\right|\right)
$$

for all $x_{1}, x_{2} \in \mathbb{R}$ and $0<t_{1}<t_{2}<T$.

Next, taking $c_{*}$ as $c_{*}=\frac{1}{2 m\left\|u_{\varepsilon}+\varepsilon\right\|_{L^{\infty}\left(Q_{T}\right)}^{m-1}}$, we have

$$
\begin{aligned}
& \partial_{x}^{2}\left(u_{\varepsilon}+\varepsilon\right)^{m-1}-c_{*} \partial_{t}\left(u_{\varepsilon}+\varepsilon\right)^{m-1}= \\
& \quad=\quad \partial_{x}^{2}\left(u_{\varepsilon}+\varepsilon\right)^{m-1}\left(1-c_{*} m\left(u_{\varepsilon}+\varepsilon\right)^{m-1}\right)-c_{*} \frac{m}{m-1}\left(\partial_{x}\left(u_{\varepsilon}+\varepsilon\right)^{m-1}\right)^{2} \\
& \quad+c_{*}(m-1)\left(u_{\varepsilon}+\varepsilon\right)^{m-2} \partial_{x}\left(\left(u_{\varepsilon}+\varepsilon\right)^{q-2} u_{\varepsilon} \partial_{x} v_{\varepsilon}\right) .
\end{aligned}
$$

As for the first term in the above, noting that $\frac{1}{2} \leq 1-c_{*} m\left(u_{\varepsilon}+\varepsilon\right)^{m-1} \leq 1$, we have by (3.23) that

$$
\partial_{x}^{2}\left(u_{\varepsilon}+\varepsilon\right)^{m-1}\left(1-c_{*} m\left(u_{\varepsilon}+\varepsilon\right)^{m-1}\right) \geq \begin{cases}0 & \text { for } \quad \partial_{x}^{2}\left(u_{\varepsilon}+\varepsilon\right)^{m-1} \geq 0 \\ -\beta & \text { for } \quad \partial_{x}^{2}\left(u_{\varepsilon}+\varepsilon\right)^{m-1}<0\end{cases}
$$

which together with (3.28) implies that there exists a positive constant $C_{3}$ depending only on $u_{0}, m, q$ and $\gamma$ such that

$$
\partial_{x}^{2}\left(u_{\varepsilon}(x, t)+\varepsilon\right)^{m-1}-c_{*} \partial_{t}\left(u_{\varepsilon}(x, t)+\varepsilon\right)^{m-1} \geq-C_{3}
$$

for all $x \in \mathbb{R}$ and $0<t<T$.

Let us now chose $R \geq 1$ arbitrary large and take the cut-off function $\hat{\eta}$ such as

$$
\hat{\eta}(x):= \begin{cases}1 & \text { for }|x|<R, \\ \exp \left(1-\frac{R}{2 R-x}\right) & \text { for } \quad R \leq|x|<2 R, \\ 0 & \text { for }|x| \geq 2 R\end{cases}
$$

for all $0 \leq t<T$. Then, it holds that

$$
\left|\partial_{x} \hat{\eta}(x)\right| \leq \frac{c}{R}, \quad\left|\partial_{x}^{2} \hat{\eta}(x)\right| \leq \frac{c}{R^{2}} \quad \text { for all } x \in \mathbb{R},
$$

where $c$ is an absolute positive constant. We have by (3.29) and (3.31) that

$$
\partial_{x}^{2}\left(\left(u_{\varepsilon}(x, t)+\varepsilon\right)^{m-1} \hat{\eta}(x)\right)-c_{*} \partial_{t}\left(\left(u_{\varepsilon}(x, t)+\varepsilon\right)^{m-1} \hat{\eta}(x)\right) \geq-C_{3}-\frac{C_{4}}{R}
$$

for all $x \in \mathbb{R}$ and $0<t<T$ with some positive constant $C_{4}=C_{4}\left(u_{0}, m, q, \gamma\right)$. 
Let us take $t_{1}$ in $(0, T)$ and introduce the following semi-linear equation with the initial time $t_{1}$ :

(H) $\begin{cases}\partial_{x}^{2} z(x, t)-c_{*} \partial_{t} z(x, t)=-2 C_{3}-\frac{C_{4}}{R}, & x \in \mathbb{R}, t>t_{1}, \\ z\left(x, t_{1}\right)=\left(u_{\varepsilon}+\varepsilon\right)^{m-1}\left(x, t_{1}\right), & x \in \mathbb{R} .\end{cases}$

Then, it holds by (3.26) that

$$
\left|z(x, t)-z\left(x_{1}, t_{1}\right)\right| \leq C_{5}\left(\left|x-x_{1}\right|+\left|t-t_{1}\right|^{\frac{1}{2}}\right)
$$

for all $x \in \mathbb{R}$ and $t_{1}<t<T$ with some positive constant $C_{5}=C_{5}\left(u_{0}, m, q, \gamma\right)$.

Now let us define the parabolic operator $\hat{P}$ on the domain $D_{R, t_{1}}$ by

$$
\hat{P}(h):=-c_{*} \partial_{t} h+\partial_{x}^{2} h+2 C_{3}+\frac{C_{4}}{R}
$$

where

$$
D_{R, t_{1}}:=\left\{(x, t) \in \mathbb{R} \times\left(t_{1}, T\right) ;-2 R<x<2 R\right\} .
$$

By (3.32) and $(\mathrm{H})$, we have

$$
\left.\hat{P}\left(\left(u_{\varepsilon}+\varepsilon\right)^{m-1} \hat{\eta}\right)\right)>0=\hat{P}(z) \quad \text { for all }(x, t) \in D_{R, t_{1}} .
$$

In addition, noting that $z\left(x, t_{1}\right)=\left(u_{\varepsilon}+\varepsilon\right)^{m-1}\left(x, t_{1}\right)$ of $(\mathrm{H})$ and $z(x, t)>0$ for all $(x, t) \in \mathbb{R} \times\left(t_{1}, T\right)$, we have that

$\left(\left(u_{\varepsilon}+\varepsilon\right)^{m-1} \hat{\eta}\right)(x, t)<z(x, t)$ for all $(x, t) \in \partial D_{R, t_{1}} \backslash\{(y, T) ;-2 R \leq y \leq 2 R\}$.

Hence, applying the comparison principle to the parabolic operator $\hat{P}$, e.g., Friedman [12, Theorem 16, page 52] or Lieberman [18, Lemma 9.4], we obtain

$$
\left(\left(u_{\varepsilon}+\varepsilon\right)^{m-1} \hat{\eta}\right)(x, t)<z(x, t) \quad \text { for all }(x, t) \in D_{R, t_{1}},
$$

which yields that

$$
\left(u_{\varepsilon}(x, t)+\varepsilon\right)^{m-1}<z(x, t) \text { for all }-R<x<R, t_{1}<t<T .
$$

Combining (3.33) with (3.34), we have by $(\mathrm{H})$ that

$$
\begin{aligned}
\left(u_{\varepsilon}\left(x_{2}, t_{2}\right)+\varepsilon\right)^{m-1}-\left(u_{\varepsilon}\left(x_{1}, t_{1}\right)+\varepsilon\right)^{m-1} & \leq z\left(x_{2}, t_{2}\right)-z\left(x_{1}, t_{1}\right) \\
& \leq C_{5}\left(\left|x_{2}-x_{1}\right|+\left|t_{2}-t_{1}\right|^{\frac{1}{2}}\right)
\end{aligned}
$$

for all $-R<x_{1}, x_{2}<R$ and $0<t_{1}<t_{2}<T$, where $C_{5}$ is the same one as in $(3.33)$. 
By virtue of (3.27) and (3.35), it holds that

$$
\begin{aligned}
& \mid u_{\varepsilon}\left(x_{2}, t_{2}\right)-u_{\varepsilon}\left(x_{1}, t_{1}\right) \mid= \\
&=\left|\left(\left(u_{\varepsilon}\left(x_{2}, t_{2}\right)+\varepsilon\right)^{m-1}\right)^{\frac{1}{m-1}}-\left(\left(u_{\varepsilon}\left(x_{1}, t_{1}\right)+\varepsilon\right)^{m-1}\right)^{\frac{1}{m-1}}\right| \\
& \leq \begin{cases}\left|\left(u_{\varepsilon}\left(x_{2}, t_{2}\right)+\varepsilon\right)^{m-1}-\left(u_{\varepsilon}\left(x_{1}, t_{1}\right)+\varepsilon\right)^{m-1}\right|^{\frac{1}{m-1}} & \text { for } m \geq 2, \\
C_{6}\left|\left(u_{\varepsilon}\left(x_{2}, t_{2}\right)+\varepsilon\right)^{m-1}-\left(u_{\varepsilon}\left(x_{1}, t_{1}\right)+\varepsilon\right)^{m-1}\right| & \text { for } 1<m<2\end{cases} \\
&(3.36) \quad \leq\left(C_{5}^{\frac{1}{m-1}}+C_{6}\right)\left(\left|x_{2}-x_{1}\right|+\left|t_{2}-t_{1}\right|^{\frac{1}{2}}\right)^{\mu}
\end{aligned}
$$

for all $-R<x_{1}, x_{2}<R$ and $0<t_{1}<t_{2}<T$ with some positive constant $C_{6}=C_{6}\left(u_{0}, m, q, \gamma\right)$, where $\mu=\min \left\{1, \frac{1}{m-1}\right\}$. This implies that $\left\{u_{\varepsilon}\right\}_{\varepsilon>0}$ is a family of equi-continuous functions in $-R<x<R$ and $0<t<T$. Hence by the Ascoli-Arzelà theorem, there is a subsequence of $\left\{u_{\varepsilon}\right\}_{\varepsilon>0}$, which we denoted by $\left\{u_{\varepsilon}\right\}_{\varepsilon>0}$ itself, such that

$$
u_{\varepsilon}(x, t) \longrightarrow u(x, t) \quad \text { as } \varepsilon \rightarrow 0
$$

uniformly in every compact set of $(-R, R) \times(0, T)$. Now letting $\varepsilon \rightarrow 0$ in (3.36), we have by (3.37) that

$$
\left|u\left(x_{2}, t_{2}\right)-u\left(x_{1}, t_{1}\right)\right| \leq\left(C_{5}^{\frac{1}{m-1}}+C_{6}\right)\left(\left|x_{2}-x_{1}\right|+\left|t_{2}-t_{1}\right|^{\frac{1}{2}}\right)^{\mu}
$$

for all $-R<x_{1}, x_{2}<R$ and $0<t_{1}, t_{2}<T$. Since $R$ can be taken arbitrary large and since the constants $C_{5}$ and $C_{6}$ are independent of $R$, we observe that

$$
\left|u\left(x_{2}, t_{2}\right)-u\left(x_{1}, t_{1}\right)\right| \leq\left(C_{5}^{\frac{1}{m-1}}+C_{6}\right)\left(\left|x_{2}-x_{1}\right|+\left|t_{2}-t_{1}\right|^{\frac{1}{2}}\right)^{\mu}
$$

for all $x_{1}, x_{2} \in \mathbb{R}$ and $0<t_{1}, t_{2}<T$. Thus we complete the proof of Theorem 2.1 (1).

Proof of Theorem 2.1 (2). By [23, Theorem 1.2 (ii)], it holds that $\partial_{x} u^{m-1+\delta}$ is a continuous function with respect to $x$ with the property that $\partial_{x} u^{m-1+\delta}(x, t)=0$ at the point $(x, t) \in \mathbb{R} \times(0, T)$ such that $u(x, t)=0$. Hence it remains to prove that $\partial_{x} u^{m-1+\delta}$ is a continuous function with respect to $t$ with the property that $\partial_{x} u^{m-1+\delta}(x, t)=0$ at the point $(x, t) \in \mathbb{R} \times(0, T)$ such that $u(x, t)=0$.

First of all, let $u\left(x_{0}, t_{0}\right)>0$. Then we see by the standard argument that $\partial_{x} u^{m-1+\delta}$ is a continuous function in a neighbourhood of $\left(x_{0}, t_{0}\right)$. Therefore, for fixed $x \in \mathbb{R}$, it suffices to prove that $\partial_{x} u^{m-1+\delta}(x, \cdot)$ is a continuous function in a neighbourhood of $t_{1}$ such as $u\left(x, t_{1}\right)=0$ with the additional 
property that $\partial_{x} u^{m-1+\delta}\left(x, t_{1}\right)=0$. Therefore, by (3.38), there exists $a_{0}>0$ such that

$$
0 \leq u(x, t) \leq\left|u(x, t)-u\left(x, t_{1}\right)\right|+u\left(x, t_{1}\right) \leq\left(C_{5}^{\frac{1}{m-1}}+C_{6}\right) a^{\mu}
$$

holds for all $t \in I_{a}\left(t_{1}\right):=\left\{t \in(0, T) ;\left|t-t_{1}\right|<a\right\}$ and for all $0<a \leq a_{0}$.

For every $\delta>0$, we have by (2.4) that

$$
\begin{aligned}
\left|\partial_{x} u^{m-1+\delta}(x, t)\right| & =\frac{m-1+\delta}{m-1}\left|u^{\delta}(x, t)\right|\left|\partial_{x} u^{m-1}(x, t)\right| \\
& \leq C a^{\mu \delta}, \quad 0<a \leq a_{0}
\end{aligned}
$$

for some positive constant $C=C\left(u_{0}, m, q, \gamma\right)$. Hence we have by letting $a \rightarrow 0$ that

$$
\partial_{x} u^{m-1+\delta}\left(x, t_{1}\right)=0
$$

which implies that $\partial_{x} u^{m-1+\delta}\left(x, t_{1}\right)$ is continuous at $t_{1}$. Since $t_{1}$ can be taken arbitrary in such a way that $u\left(x, t_{1}\right)=0$, we conclude that $\partial_{x} u^{m-1+\delta}\left(x, t_{1}\right)$ is a continuous function in $(0, T)$ for all $x \in \mathbb{R}$ with the additional property that $\partial_{x} u^{m-1+\delta}(x, t)=0$ for the point $(x, t)$ such as $u(x, t)=0$.

Concerning $1<m<2$, by [23, Theorem 1.2 (ii)], it holds that $\partial_{x} u$ is a continuous function with respect to $x$ with the property that $\partial_{x} u(x, t)=0$ at the point $(x, t) \in \mathbb{R} \times(0, T)$ such that $u(x, t)=0$. Hence it remains to prove that $\partial_{x} u$ is a continuous function with respect to $t$ with the property that $\partial_{x} u(x, t)=0$ at the point $(x, t) \in \mathbb{R} \times(0, T)$ such that $u(x, t)=0$. This can be handled in a similar manner as above. Indeed, we conclude that $\partial_{x} u\left(x, t_{1}\right)$ is a continuous function at $t_{1}$ for all $x \in \mathbb{R}$ with the additional property that $\partial_{x} u(x, t)=0$ for the point $(x, t)$ such as $u(x, t)=0$. Thus we complete the proof of Theorem 2.1 (2).

\subsection{Proof of Theorem 2.2}

By (3.2), (3.37) and the weakly-star compactness of $L^{\infty}(\mathbb{R} \times(0, T))$, there exists a sequence of $\left\{u_{\varepsilon}\right\}_{\varepsilon>0}$, which we denote by $\left\{u_{\varepsilon}\right\}_{\varepsilon>0}$ itself for simplicity, such that

$$
\partial_{x}\left(u_{\varepsilon}+\varepsilon\right)^{m-1} \rightarrow \partial_{x} u^{m-1} \quad \text { weakly }- \text { star } \quad \text { in } L^{\infty}(\mathbb{R} \times(0, T)) .
$$

This together with (3.3) in Lemma 3.2 yields that

$$
\int_{0}^{T} \int_{\mathbb{R}}\left(\beta \varphi-\partial_{x} u^{m-1} \cdot \partial_{x} \varphi\right) d x d t \geq 0
$$

for all $\varphi \in C_{0}^{1}(\mathbb{R} \times(0, T))$ with the property $\varphi(x, t) \geq 0$ for all $(x, t) \in$ $\mathbb{R} \times(0, T)$. Thus we complete the proof of Theorem 2.2 . 


\subsection{Proof of Theorem 2.3}

The proof of Theorem 2.3 is based on the standard argument. For reader's convenience, we shall give the proof.

It holds by (3.21) that

$$
\partial_{t} u_{\varepsilon}(x, t) \geq-C\left(u_{\varepsilon}(x, t)+\varepsilon\right),
$$

which yields that

$$
\partial_{t}\left(\log \left(u_{\varepsilon}(x, t)+\varepsilon\right)\right) \geq-C
$$

for all $x \in \mathbb{R}$ and $0<t<T$ with some positive constant $C=C\left(u_{0}, m, q, \gamma\right)$ independent of $0<\varepsilon<1$. Integrating both sides with respect to $t$ from $t_{0}$ to $t$, we have that

$$
\log \left(\frac{u_{\varepsilon}(x, t)+\varepsilon}{u_{\varepsilon}\left(x, t_{0}\right)+\varepsilon}\right) \geq-C\left(t-t_{0}\right)=\log e^{-C\left(t-t_{0}\right)}
$$

for all $x \in \mathbb{R}$ and $0<t_{0}<t<T$. This implies that

$$
u_{\varepsilon}(x, t)+\varepsilon \geq\left(u_{\varepsilon}\left(x, t_{0}\right)+\varepsilon\right) e^{-C\left(t-t_{0}\right)}
$$

for all $x \in \mathbb{R}$ and $0<t_{0}<t<T$. Now, letting $\varepsilon$ tend to 0 in (3.39), we have by (3.37) that

$$
u(x, t) \geq u\left(x, t_{0}\right) e^{-C\left(t-t_{0}\right)}
$$

for all $x \in \mathbb{R}$ and $0<t_{0}<t<T$, which yields the desired result. This completes the proof of Theorem 2.3.

The proof of Corollary 2.4 is obvious, so we may omit it.

Acknowledgments: The author would like to express her sincere gratitude to Professors H. Kozono and T. Nagai for many stimulating conversation and helpful advice.

\section{References}

[1] Aronson, D. G.: The porous medium equation. In Nonlinear diffusion problems (Montecatini Terme, 1985), 1-46. Lecture Notes in Math. 1224. Springer, Berlin, 1986.

[2] Aronson, D. G.: Regularity properties of flows through porous media: A counterexample. SIAM J. Appl. Math. 19 (1970), 299-307.

[3] Aronson, D. G.: Regularity properties of flows through porous media: The interface. Arch. Rational Mech. Anal. 37 (1970), 1-10. 
[4] Aronson, D. G. and Bénilan, P.: Régularité des solutions de l'équation des milieux poreux dans $\mathbb{R}^{N}$. C.R. Acad. Sci. Paris Sér. A-B 288 (1979), A103-A105.

[5] Aronson, D. G. and Caffarelli, L. A.: Optimal regularity for one dimensional porous medium flow. Rev. Mat. Iberoamericana 2 (1986), no. 4, $357-366$.

[6] BÉnilan, Ph.: A strong regularity $L^{p}$ for solutions of the porous media equation. In Contributions to nonlinear partial differential equations (Madrid, 1981), 39-58. Res. Notes in Math. 89. Pitman, Boston, MA, 1983.

[7] Caffarelli, L. A. and Friedman, A.: Continuity of the density of a gas flow in a porous medium. Trans. Amer. Math. Soc. 252 (1979), 99-113.

[8] Childress, S. And Percus, J. K.: Nonlinear aspects of chemotaxis. Math. Biosci. 56 (1981), 217-237.

[9] Dahlberg, B. E. J. And Kenig, C. E.: Nonnegative solutions of the porous medium equations. Comm. Partial Differential Equations 9 (1984), 409-437.

[10] DiBenedetto, E.: Regularity results for the porous medium equation. Ann. Mat. Pura Appl. (4) 121 (1979), 249-262.

[11] DiBenedetto, E. And Friedman, A.: Hölder estimates for nonlinear degenerate parabolic systems. J. Reine Angew. Math. 357 (1985), 1-22.

[12] Friedman, A.: Partial differential equations of parabolic type. PrenticeHall, Englewood Cliffs, NJ, 1964.

[13] Gilding, B. H.: Hölder continuity of solutions of parabolic equations. J. London Math. Soc. (2) 13 (1976), 103-106.

[14] Gilding, B. H. and Peletier, L. A.: On a class of similarity solutions of the porous medium equation. J. Math. Anal. Appl. 55 (1976), 351-364; J. Math. Anal. Appl. 57 (1977), 522-538.

[15] Keller, E. F. And Segel, L. A.: Initiation of slime mold aggregation viewed as an instability. J. Theor. Biol. 26, (1970), 399-415.

[16] Knerr, B.: The porous medium equation in one dimension. Trans. Amer. Math. Soc. 234 (1977), no. 2, 381-415.

[17] Kruzhkov, S. N.: Results on the character of the regularity of solutions of parabolic equations and some of their applications. Math. Notes 6 (1969), $517-523$.

[18] Lieberman, G. L.: Second order parabolic differential equations. World Scientific Publishing, River Edge, NJ, 1996.

[19] Luckhaus, S. And Sugryama, Y.: Asymptotic profile with the optimal convergence rate for a parabolic equation of chemotaxis in super-critical cases. Indiana Univ. Math. J. 56 (2007), 1279-1297.

[20] Sugiyama, Y.: Time global existence and asymptotic behavior of solutions to degenerate quasi-linear parabolic systems of chemotaxis. Differential Integral Equations 20 (2007), 133-180. 
[21] Sugryama, Y.: Application of the best constant of the Sobolev inequality to degenerate Keller-Segel models. Adv. Differential Equations 12 (2007), $121-144$

[22] Sugiyama, Y. And Kunit, H.: Global existence and decay properties for a degenerate Keller-Segel model with a power factor in drift term. J. Differential Equations 227 (2006), 333-364.

[23] Sugryama, Y.: Finite speed of propagation in 1-D degenerate Keller-Segel system. To appear in Math. Nachr.

[24] Sugiyama, Y.: Interfaces for 1-D degenerate Keller-Segel systems. J. Evol. Equ. 9 (2009), no. 1, 123-142.

[25] Sugiyama, Y. And Velázquez, J. J.L.: Self-similar aggregation with different masses for the Keller-Segel system with porous medium diffusion. Submitted.

[26] VÁzquez, J. L.: The porous medium equation. Mathematical Theory. Oxford Mathematical Monographs. The Clarendon Press, Oxford University Press, Oxford, 2007.

Recibido: 3 de diciembre de 2008

Yoshie Sugiyama

Department of Mathematics

Tsuda University

2-1-1, Tsuda-chou, Kodaira-shi Tokyo, 187-8577, Japan sugiyama@tsuda.ac.jp 